
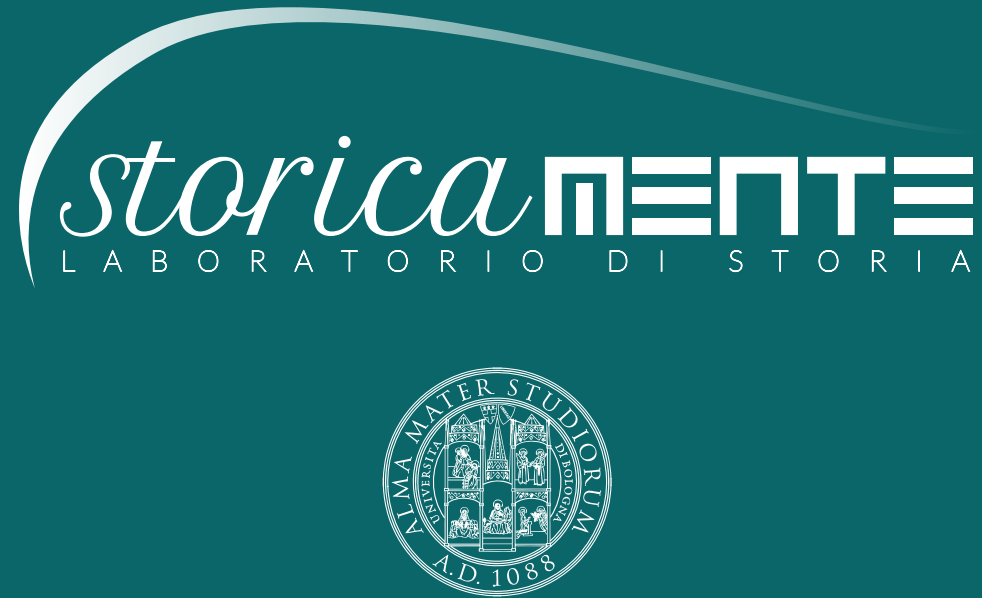

ALMA MATER STUDIORUM

Università di Bologna

Dipartimento di Storia Culture Civiltà

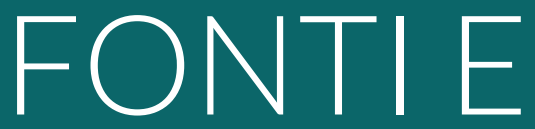




\section{STORICAMENTE.ORG}

\section{Laboratorio di Storia}

Luca Tedesco

La sicurezza nelle miniere sarde tra Otto e Novecento: cosa ci raccontano le statistiche

Numero $14-2018$

ISSN: $1825-411 \mathrm{X}$

Art. 45

pp. 1-2

DOI: $10.12977 /$ stor735

Editore: BraDypUS

Data di pubblicazione: 22/03/2019

Fonti e documenti: Frammenti di storia del lavoro nelle miniere sarde: tra archivi e memoria, a cura di Luca Tedesco 


\title{
Frammenti di storia del lavoro nelle miniere sarde: tra archivi e memoria. Introduzione
}

\author{
LUCA TEDESCO \\ Univ. Roma Tre, Dipartimento di \\ Scienze della Formazione
}

Il processo di deindustrializzazione conosciuto dal comparto minerario sardo affonda le radici negli anni Sessanta, quando la crisi delle principali società minerarie private portò all'acquisizione, a partire dal 1968, dei siti minerari da parte di compagnie a gestione statale. Negli anni Settanta, il comparto fu investito da un vero e proprio processo di deindustrializzazione con la contrazione e successiva chiusura dei principali siti minerari ancora attivi nel corso degli anni Ottanta. Nell'arco di poco meno di un trentennio, si assistette a un calo verticale degli occupati. Nel 1997 venne decretata la definitiva chiusura dei siti ancora attivi, all'epoca gestiti da società a partecipazione statale e regionale. Il forte impatto sulle comunità dell'esaurirsi dell'attività mineraria nel corso degli anni Novanta produsse la mobilitazione della cittadinanza per la rigenerazione del territorio, che, nel caso sardo, portò alla creazione alla fine del decennio del Parco geominerario storico e ambientale della Sardegna, riconosciuto dall'Unesco quale primo parco della futura rete mondiale dei geositi/geoparchi dell'Unesco.

Negli anni Duemila, si è registrata un'intensa attività di recupero delle fonti delle società minerarie, attività che ha dato vita a importanti ar- 
chivi, anche di valenza internazionale, come l'Archivio storico minerario Igea Spa, a tutt'oggi non sufficientemente esplorati.

I contributi del dossier "Frammenti di storia del lavoro nelle miniere sarde: tra archivi e memoria" vanno allora concepiti come frammenti, tasselli di un più ampio disegno volto a valorizzare tali fonti, disegno ancora in larga parte da eseguire.

Se da una parte le video-interviste a ex minatori e cernitrici, realizzate su sollecitazione dell'Archivio storico minerario Igea, hanno reso possibile la ricostruzione della cultura novecentesca del lavoro nei suoi aspetti tecnici, delle condizioni di lavoro e della loro evoluzione nel tempo (Azara, Betti), oltre che dei background familiari, dall'altra fonti a stampa, resoconti, relazioni e rapporti prodotti dalle società minerarie e da diversi soggetti pubblici hanno dato conto degli esperimenti posti in essere già negli Venti per incrementare la produzione e quindi ridurre gli infortuni (Cuccu).

A proposito di questi ultimi, infine, è stata avanzata l'ipotesi che la lettura fornita dalla storiografia e dalla memorialistica in materia di sicurezza nelle miniere sarde nell'Italia della seconda rivoluzione industriale, lettura incupita, a tinte fosche, che fa della miniera un luogo fortemente insicuro, segnato da uno stillicidio di tragedie e infortuni mortali, non sia suffragata dai dati statistici disponibili (Tedesco). 